\title{
Cut-paste string operation for collaborative groupware applications
}

\author{
Santosh Kumawat ${ }^{1^{*}}$ (D) and Ajay Khunteta ${ }^{2}$
}

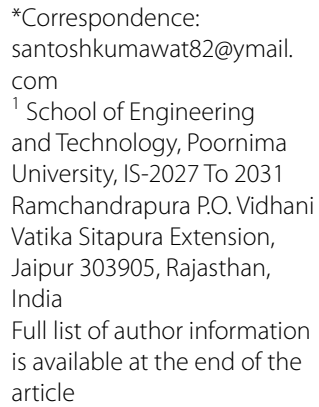

\begin{abstract}
Operational transformation (OT) is a concurrency control approach in multi-user collaborative groupware system. With OT, local operations do not delay and shared document could be edited by users at any time. Most of OT algorithms cannot be formally proved. Till date only two character-wise primitive operations Insert and Delete are proposed and these operations have time complexity of the order of $\mathrm{O}\left(|\mathrm{H}|^{2}\right)$ where $\mathrm{H}$ is the history buffer of operations. In this paper, a new algorithm for cut-paste string operation is proposed with time complexity and space complexity of the order of $\mathrm{O}(|\mathrm{H}|)$. It reduces the time and space consumption in performing composite string operation-Cut-Paste. The proposed algorithm also handles overlapping and splitting of operations. A modified algorithm GSMRITFDD is also proposed that has removed all faults of existing ITDD algorithm.
\end{abstract}

Keywords: Operational Transformation Algorithms, Concurrency control, Distributed systems, Collaborative system, Groupware system

\section{Background}

Groupware system is a multiple user system in which the operations of each user must quickly be propagated to all other shared users (e.g., multi-player game, real-time computer conferencing).

Groupware system requires sharing of data, fine granularity, concurrency control and fast response times. Consistency and high local responsiveness are specific requirements for multi-user systems (Ellis and Gibbs 1989; Sun et al. 1998). Concurrency control protocols are used to remove inconsistency in the multi-user transactions systems such as Relational Databases, Distributed Systems and Groupware Systems.

Theorem 1 In a consistent shared environment which has replicated data after execution of all operations all have same data.

Traditional concurrency control methods such as Locking and Reversible Execution may cause the loss of some user interaction results. These methods are not suitable for distributed interactive applications which require fast local response satisfying user intentions, intention consistency and convergence. In past 15 years, OT is an acceptable method for consistency and concurrency maintenance in multi-user group editors such

\section{Springer}

(c) 2015 Kumawat and Khunteta. This article is distributed under the terms of the Creative Commons Attribution 4.0 International License (http://creativecommons.org/licenses/by/4.0/), which permits unrestricted use, distribution, and reproduction in any medium, provided you give appropriate credit to the original author(s) and the source, provide a link to the Creative Commons license, and indicate if changes were made. 
as group editors and Google Wave1 (Davis et al. 2002; Ellis and Gibbs 1989; Sun et al. $1998 \mathrm{a}, \mathrm{b})$. When compared to other concurrency control methods, OT achieves convergence, causality and intention preservation without sacrificing local responsiveness and concurrent work (Sun et al. 1998a). OT allows users to edit any part of the data which is shared by other users (Bentley and Dourish 1995).

\section{Review of OT algorithms}

In last 25 years of OT algorithms, there are two main challenges in OT: First, most of algorithms require an informal condition known as "Intention Preservation" and their correctness cannot be formally proved. Second: except for (Ressel et al. 1996), all existing OT algorithms only support two character-based operations called insert and delete.

The proposed work studied major OT algorithms (Table 1) including Distributed Operation Transformation (dOPT) algorithm (Ellis and 1989), the Generic Operational Transformation (GOT) algorithm (Sun et al. 1998), GOT Optimized (GOTO) algorithm (Sun et al. 1998), State Difference Transformation (SDT) algorithm (Li and Li 2007), SCOT2 (Suleiman et al. 1998), SCOT 3/4 algorithm (Vidot et al. 2000), Adopted (adOPTed) algorithm (Ressel et al. 1996), Admissibility-Based Transformation (ABT) algorithm (Li and Li 2010), ABTUndo (ABTU) Algorithm ( $\mathrm{Li}$ and Li 2007), admissibility-based sequence transformation (ABST) (Shao et al. 2010a, b), and Admissibility-Based Transformation with Strings (ABTS) algorithm (Shao et al. 2009).

The ABT (Li and Li 2007) introduces a correctness criterion called admissibility preservation, in which correctness of admissibility-based transformation (ABT) functions has formally proved. ABTU arranges the operation history in total effects-relation order and improves the time complexity to $\mathrm{O}(\mathrm{IHI})$. In the available literature, only GOT, GOTO and ABTS algorithms support string-wise operations. The time complexity of GOT and GOTO algorithms are $\mathrm{O}\left(\left|\mathrm{H}^{2}\right|\right)$ but time complexity of ABTS is only $\mathrm{O}(|\mathrm{H}|)$. So due to less time complexity, ABTS is better string-based OT algorithm as compared to GOT and GOTO. The proposed work is focused on string-based OT algorithm which is based on ABT framework.

\section{OT framework}

Most of existing OT algorithms have developed under a well-accepted framework with a condition that algorithm cannot be formally proved. In addition, they generally support two character-based primitive operations like insert and delete in a linear data structure. Only three algorithms called as GOT, GOTO and ABTS support primitive string operations. This research paper proposes a new novel OT algorithm for composite string operation Cut-Paste with existing primitive operations.

The insert(p, s) and delete(p, s), insert and delete a string 's' at position ' $\mathrm{p}$ ' in the shared data, respectively. The proposed work has introduced a new composite string operation Cut-Paste (p1, s, p2) which cut from position 'p1' and paste it at position 'p2' in shared data with less time complexity.

In OT, shared data are like a linear string 's' of atomic characters in which objects are referred by their positions 'p' starting from zero in the string and consider two only primitive string operations, called, insert(p, s) and delete(p, s). At site, there exists a common definition state 's' for all operations. The standard notations are summarized in Table 2 (Shao et al. 2009). 


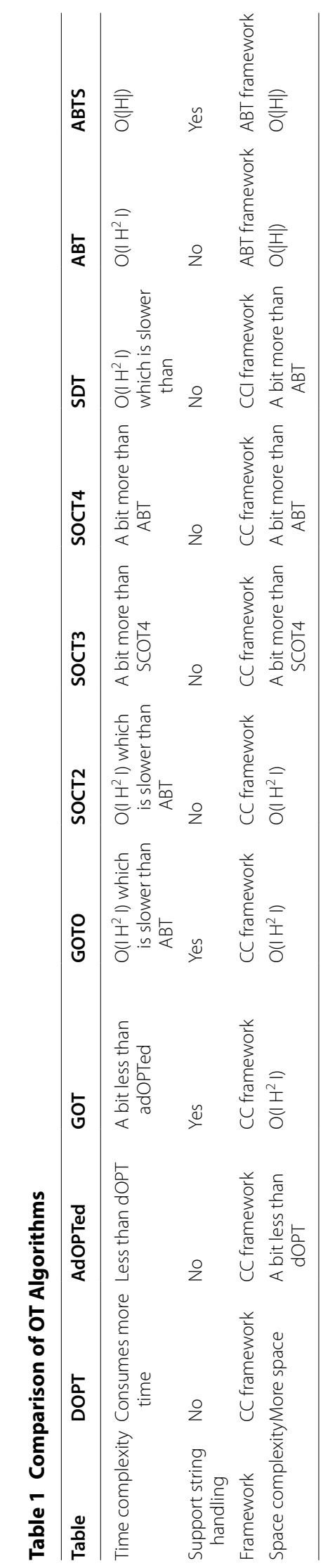


Table 2 Standard Notations

\begin{tabular}{|c|c|}
\hline Notations & Description \\
\hline o.id & Id of site that generates operation o \\
\hline o.type & Type of operation o, i.e., either insert or delete \\
\hline o.pos & Position of operation o \\
\hline O.str & String insert/delete by o \\
\hline $\mathrm{o} 1 \rightarrow \mathrm{o} 2$ & o1 occurs before o2 \\
\hline $01 \| 02$ & 01 and $\mathrm{o} 2$ are concurrent \\
\hline o1Uo2 & 01 and $\mathrm{o} 2$ are contextually equivalent \\
\hline $\mathrm{O} 1 \rightarrow \mathrm{O} 2$ & 01 and 02 are contextually serialized \\
\hline$[01,02]$ & An ordered list of two operations o1 and o2 \\
\hline$<01,02>$ & Two operations in sequence \\
\hline$|\mathrm{L}|$ & Number of objects in list L \\
\hline L1.L2 & Concatenation of two lists L1 and L2 \\
\hline s[i:len] & Substring of string s starts from position i of length len \\
\hline $\mathrm{Sq}$ & A sequence is a special list \\
\hline $\mathrm{R} 1=[01$. start, o1.end $]$ & Operation region of operation o1 is $\mathrm{R} 1$ which starts from o1.start \& ends at o1.end \\
\hline cut-paste(p1, s, p2) & Cut a string s from $\mathrm{p} 1$ and paste at p2 \\
\hline exec(oi) & Execution of operation oi \\
\hline
\end{tabular}

\section{Methods}

The proposed algorithm for cut-paste string composite operation ITCutPaste (Inclusive Transformation of Cut-Paste) is based on inclusive transformation of operational transformation. The algorithm ABTS (Shao et al. 2009), already contains ITII (Inclusive Transformation of Insert-Insert)/ITID (Inclusive Transformation of Insert-Delete)/ ITDD (Inclusive Transformation of Delete-Delete)/ITDI (Inclusive Transformation of Delete-Insert) algorithms for transformation of insert and delete string operations. So to perform cut-paste operation, first the substring ' $\mathrm{s}$ ' get deleted from $\mathrm{p}$ by operation delete(s, p) and then 's' get inserted at position q by operation insert(s, q). Here, delete(s, p) and insert(s, q) are independent operations. At all sharing sites, transformation functions corresponding to both delete(p, s) and insert $(\mathrm{q}, \mathrm{s})$ get called which results in increase in the time complexity.

This research work proposes a single transformation algorithm for composite string operation cut-paste. So all sites require only single transformation function for cut-paste operation. The time complexity of transformation function ITCutPaste for cut-paste operation is same as transformation function for insert or delete ITDI/ITID. It is based on inclusive transformation and ABT Framework so it can be formally proved.

\section{Type of operations}

ITCutPaste consists of operations $\mathrm{o} 1, \mathrm{o} 2$ as input and $\mathrm{o} 1^{\prime}$ as output. Operations $\mathrm{o} 1, \mathrm{o} 2$ and $\mathrm{o} 1^{\prime}$ may be string or character operations which operate on shared data in multiuser groupware collaborative applications like group editors.

Definition 1 o1 and o2 are contextually equivalent o1 ||o2, o1Uo2 and if input is o1 and output then output should be $\mathrm{o} 2 \rightarrow \mathrm{o} 1^{\prime}$. 


\section{Design and analysis of algorithms}

ITCutPaste consists many sub-algorithms ITDCp (Inclusive Transformation of Deletion with Cut-Paste), ITICp (Inclusive Transformation of Insertion with Cut-Paste), ITCpI (Inclusive Transformation of Cut-Paste with Insertion), and ITCpD (Inclusive Transformation of Cut-Paste with Deletion). The proposed algorithm satisfies causality and admissibility preservation.

Definition 2 If we have $\operatorname{exec}\left(\mathrm{o}_{\mathrm{i}}\right)$, then all exec $\left(\mathrm{o}_{\mathrm{i}-1}\right)$ must be completed then only oi satisfies causality.

Definition 3 If o1Uo2 then IT $(01,02)$ satisfies admissibility. It does not have inconsistent order at shared environment.

Theorem 2 Let $\mathrm{H}$ is admissible history of operation which satisfies causality. Also, sq is a sequence of operations and $\mathrm{sq}_{\sim}^{\sim} \mathrm{H}$. Then, if o is executed in state $\mathrm{s}$, we have exec(s, H). There should be $\mathrm{sq}^{\prime \sim} \sim(\mathrm{sq} . \mathrm{o})$ and $\mathrm{sq}^{\prime}$ must be consistent.

The ITCutPaste(o1, o2) transforms o1 with another operation o2 with output of this function is $\mathrm{ol}^{\prime}$. The precondition of ITCutPaste(o1, o2) is o1Uo2 and the post-condition is $\mathrm{o} 2 \rightarrow \mathrm{o} 1$ '. The following "Type of operations" presented the algorithm ITDCp and "Design and analysis of algorithms" presented the algorithm ITICp. In algorithm ITCutPaste if o1 operation type is insert and o2 operation type is CutPaste then ITICp is called and if 01 operation type is delete and $\mathrm{o} 2$ operation type is CutPaste then ITDCp is called. If 01 operation type is CutPaste and o 2 operation type is insert, then ITCPI is called and if o1 operation type is CutPaste and o 2 operation type is delete then ITCpD is called.

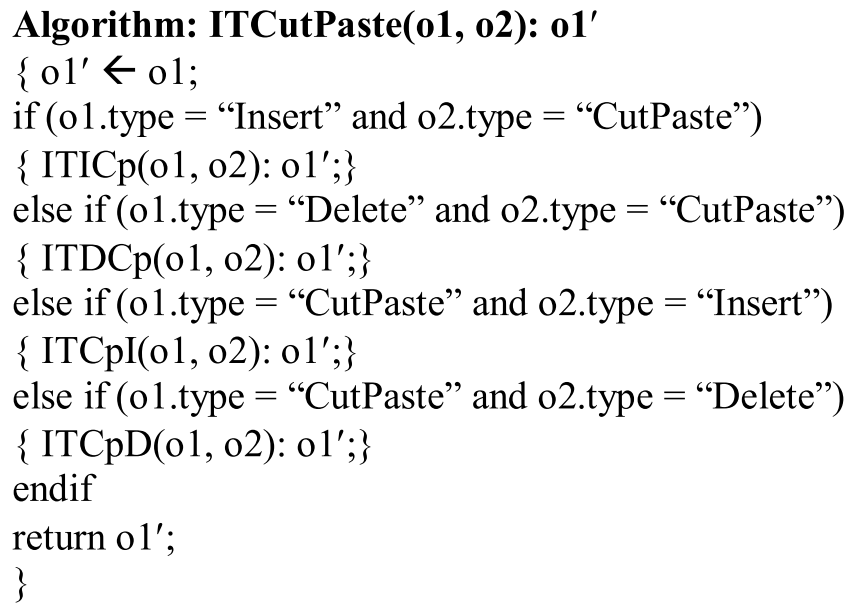

\section{Algorithm ITDCp}

Algorithm ITDCp takes as parameters o1 and o2 and return $\mathrm{o} 1^{\prime}$. Here, $\mathrm{o} 1$ is deletion operation and $\mathrm{o} 2$ is Cut-Paste operation. Here, precondition is $01 \mathrm{Uo} 2$ and post-condition is $\mathrm{o} 2 \rightarrow \mathrm{o} 1^{\prime}$. GSMRITFDD(o1, o2) used in Algorithm ITDCp is transformation algorithm to transform o1 (delete) and o2 (delete) operations. 


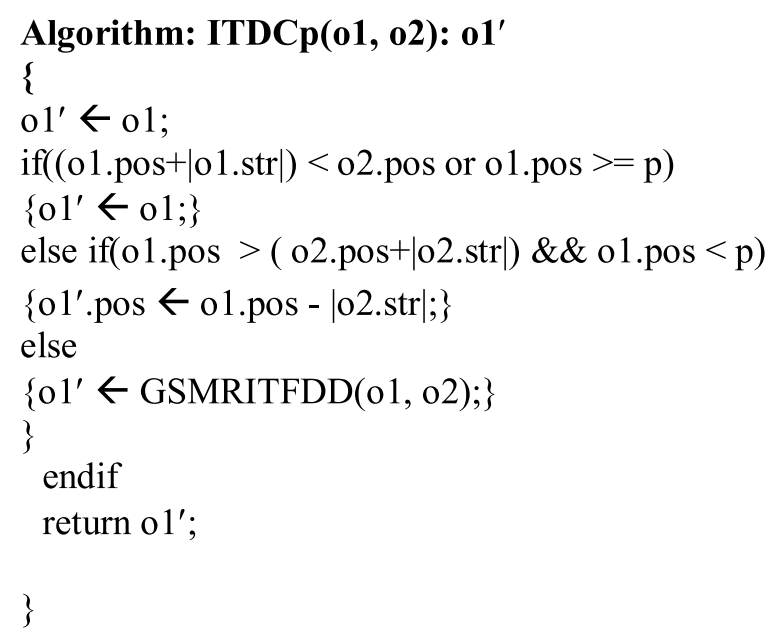

\section{Algorithm ITICp}

Algorithm ITICp takes as parameters 01 and $o 2$ and return $o 1^{\prime}$. Here, o1 is insertion operation and $\mathrm{o} 2$ is Cut-Paste operation. Here, precondition is o1Uo2 and post-condition is $02 \rightarrow \mathrm{O}^{\prime}$.

Algorithm: ITICp (o1, o2): o1'

\{

$\mathrm{o} 1^{\prime} \leftarrow \mathrm{o} 1$

if $(\mathrm{o} 1 . \operatorname{pos}<=02$. pos \&\& o1 $>=$ p)

$\left\{01^{\prime} \leftarrow 01 ;\right\}$

elseif(o1 $>=02 . p o s \& \&$ o $1<=02 \cdot$ pos $+|02 . s t r|)$

$\left\{01^{\prime} \cdot\right.$ pos $=02 \cdot$ pos; $\}$

elseif(o1.pos $>$ (o2.pos $+\mid$ o2.str $\mid \& \&$ o $1<\mathrm{p})$

$\left\{\mathrm{ol}^{\prime} \cdot\right.$ pos $=$ o1.pos $\left.-|\mathrm{o} 2 . \mathrm{str}| ;\right\}$

\}

\section{Algorithm GSMRITFDD}

The new algorithm GSMRITFDD is proposed that removed all faults of existing ITDD (Shao et al. 2009) for inclusive transformation of two deletions and work well in all possible cases. It needs to consider the following cases regarding the relations between the two target regions, $\mathrm{R} 1=\mathrm{s}[\mathrm{o} 1 \cdot \mathrm{pos}:(|\mathrm{o} 1 . \mathrm{str}|+\mathrm{o} 1 . \mathrm{pos})]$ and $\mathrm{R} 2=\mathrm{s}[\mathrm{o} 2 . \mathrm{pos}:(\mid \mathrm{o} 2$. $\operatorname{pos}|+|$ o2.str $\mid)]$ 


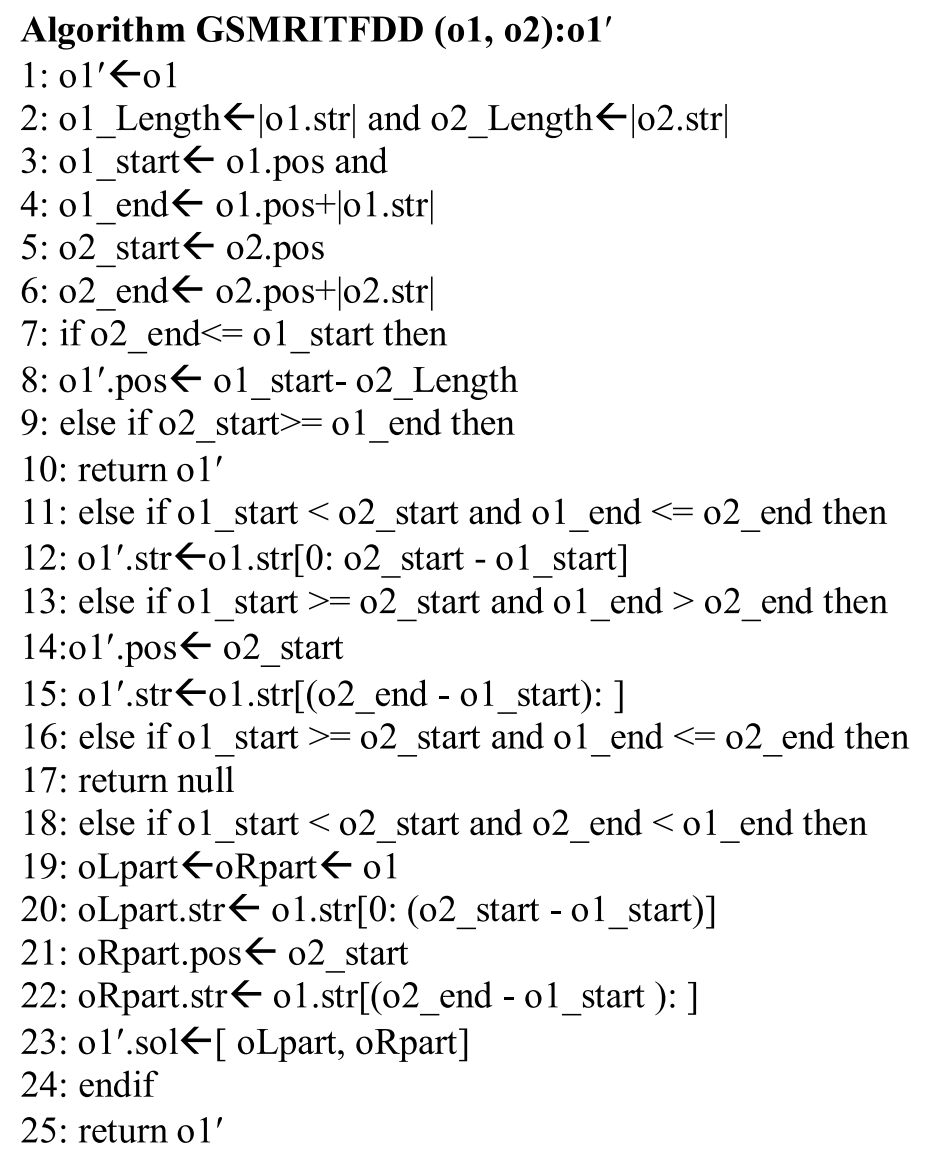

Consider the following cases regarding the relations between the two target regions, $\mathrm{R} 2=\mathrm{s}$ [o2.pos: (o2.pos + lo2.strl)] and R1 = s [o1.pos: (lo1.strl + o1.pos)].

1. (By line-9) When $R 2$ is completely on the right of R1. In this case, Deletion of R2 does not affect 01 and hence $o 1$ is returned as-is.

2. (By line-7) If $\mathrm{R} 1$ is on the right of R2. In this case, after $\mathrm{R} 2$ is deleted, we shift $\mathrm{o} 1^{\prime}$.pos by 1o2.strl characters to the left to get transformed string operation $\mathrm{o} 1^{\prime}$.

3. (By line-16) R1 is included in R2. In this case, after o2 is executed, R1 is already deleted. So there is no longer need to execute o1. That is why it returns an empty operation 4 .

4. (By line-13) When R2 partially overlaps with R1 around the left border of R1. After o2 is executed, the left part of R1 is already deleted. Hence, in this case, we need to reset o1.pos so that it will start from (o2.pos). So o1.str only needs to include the right part that is not deleted by o2, starting from (o2.pos + lo2.strl)-o1.pos in the original o1.str.

5. (By line-11) When R2 partially overlaps with R1 around the right border of R1. In this case, this is similar to case (4). After o2 is executed, o1 only needs to delete the left part that is not deleted by 02 .

6. (By line-18) R2 is included in R1. The deletion of $\mathrm{R} 2$ within $\mathrm{R} 1$ divides $\mathrm{R} 1$ into three parts, among which the middle overlapping part is already deleted by 02 . Hence, o1 
must be split into two sub-operations that delete the two remaining substrings left and right, respectively.

\section{Result and discussion}

We have implemented ABTS and ITCutPaste in lab using Qualnet and ASP.Net software in multi-user environment. The Benchmark Dataset of online Group Editor is used to verify it. We have implemented existing IT algorithm ABTS and our proposed algorithm ITCutPaste to perform string composite operation cut-paste. We experimentally conclude that ABTS consumed more time by a factor in multiple of $|\mathrm{H}|$ as compared to ITCutPaste. ITCutPaste satisfies causality preservation and admissibility preservation.

\section{Correctness proof}

If we use ABTS to transform cut-paste string operation and if $01=$ "insert" and o2 = "CutPaste", then we need ITID and ITII both for it. ITID applies for all o2.pos $\leq$ (o1.pos $+\mid$ o1.str $\mid)$ and ITII for all o2.pos $\leq$ o1.pos. Now, ITICp apply only for (o1.pos > o2.pos \&\& o1.pos $<$ p). Time complexity of ITII, ITID and ITICp is of same order. Since ITICp is for less number of operations in less range, so it consumes less or equal time as compared to ITID/ITII. ITICp could not consume more time as compared to ABTS(ITID + ITII) to operate same cut-paste string operation.

If we use ABTS to transform cut-paste string operation and if $\mathrm{ol}=$ "delete" and o2 = "CutPaste", then we need ITDI and ITDD both for it. ITDI applies for all o2.pos $<$ (o1.pos $+\mid$ o1.str $\mid)$ and ITDD for all o1.pos $>=($ o2.pos $+\mid$ o2.str $\mid)$. Now, ITDCp apply only for (o1.pos > o2.pos \&\& o1.pos < p). Time complexity of ITDD, ITDI and ITDCp is of same order. Since ITDCp is for less number of operations in less range, so it consumes less or equal time as compared to ITDI/ITDD. ITDCp could not consume more time as compared to ABTS (ITDI +ITDD) to operate same cut-paste string operation.

Similarly, time complexity of ITCpD is of same order of ITDD/ITID. But to transform o2 = "delete" and $\mathrm{o} 1=$ "CutPaste" in case of ABTS, both ITDD and ITID need to get called for greater range of operations but for ITCutPaste only ITCpD is sufficient for same or less range of operations as compared to either ITDD or ITID of ABTS. Also, time complexity of ITCPI is of same order of ITDI/ITII. But to transform o2 = "insert" and $\mathrm{o} 1=$ "CutPaste" in case of ABTS, both ITDI and ITII need to get called but for ITCutPaste only ITCPI is sufficient for same or less range of operations as compared to either ITDI or ITII of ABTS. So ITCutPaste is more efficient.

ABTS time consumption for string operation cut-paste is if $2 \mathrm{O}(|\mathrm{H}|)$ then ITCutPaste consumes only $\mathrm{O}(|\mathrm{H}|)$ time and space complexity.

A graphical representation of ABTS and ITCutPaste time complexity for various cases is shown below.

\section{Conclusion}

OT is the best method for concurrency and consistency control in multi-user groupware systems. Most of OT algorithms support character operations and very few support string primitive operations like insert and delete. The proposed algorithm ITCutPaste for string composite operation cut-paste works well in all conditions and handles 
overlapping of operations. It is based on ABT framework and can be formally proved. It has time complexity and space complexity $\mathrm{O}(|\mathrm{H}|)$ (where $\mathrm{H}$ is history buffer) similar to ABTS. The proposed algorithm GSMRITFDD has removed all faults of existing ITDD for inclusive transformation of two deletions and works well in all possible cases.

\section{Authors' contributions}

SK made substantial contributions to conception and design, acquisition of data, and analysis and interpretation of data; has been involved in drafting the manuscript or revising it critically for important intellectual content; has given final approval of the version to be published, and agrees to be accountable for all aspects of the work in ensuring that questions related to the accuracy or integrity of any part of the work are appropriately investigated and resolved. AK provided full guidance and support in all of the above works. Both authors read and approved the final manuscript.

\section{Author details}

${ }^{1}$ School of Engineering and Technology, Poornima University, IS-2027 To 2031 Ramchandrapura P.O. Vidhani Vatika Sitapura Extension, Jaipur 303905, Rajasthan, India. ${ }^{2}$ Department of Computer Science and Engineering, Poornima College of Engineering, Jaipur, India.

\section{Competing interests}

The authors declare that they have no competing interests.

Received: 6 July 2015 Accepted: 10 October 2015

Published online: 23 November 2015

\section{References}

Bentley R, Dourish P (1995) Medium versus mechanism: Supporting collaboration through customization. In: ECSCW'95 Proceedings

Davis AH, Sun C, Lu J (2002) Generalizing operational transformation to the standard general markup language. In: Proceedings of ACM Conference Computer-Supported Cooperative Work (CSCW'02), pp 58-67

Ellis CA, Gibbs SJ (1989) Concurrency control in groupware systems. In: ACM SIGMOD'89 Proceedings, Portland Oregon, pp 399-407

Li R, Li D (2007) A new operational transformation framework for real-time group editors. IEEE Trans Parallel Distrib Syst 18(3):307-319

Li D, Li R (2010) An admissibility-based operational transformation framework for collaborative editing systems. In: Computer Supported Cooperative Work (2010) 19:1-43, Springer 2009. doi:10.1007/s10606-009-9103-1

Ressel M, Nitsche-Ruhland D, Gunzenha"user R (1996) An integrating, transformation-oriented approach to concurrency control and undo in group editors. In: Proceedings ACM Conference on Computer-Supported Cooperative Work (CSCW'96), pp 288-297

Shao B, Li D, Gu N (2009) ABTS: a transformation-based consistency control algorithm for wide-area collaborative applications. In: 5th International Conference on Collaborative Computing: Networking, Applications and Worksharing. Collaborate Com 2009. Vol, 1, No. 10, pp 11-14. doi:10.4108/ICSTCOLLABORATECOM2009.8271

Shao B, Li D, Gu N (2010a) An algorithm for selective undo of any operation in collaborative applications. In: ACM

Shao B, Li D, Gu N (2010b) A Fast operational transformation algorithm for mobile and asynchronous collaboration. IEEE Trans Parallel Distrib Syst 21(12)

Suleiman M, Cart M, Ferrié J (1998) Concurrent operations in a distributed and mobile collaborative environment. In: Proceedings of the Fourteenth International Conference on Data Engineering, February. pp 23-27

Sun C, Ellis C (1998) Operational transformation in real-time group editors: issues, algorithms, and achievements. In: ACM CSCW'98, pp 59-68

Sun C, Jia X, Zhang Y, Yang Y, Chen D (1998) Achieving convergence, causality-preservation, and intention preservation in real-time cooperative editing systems. ACM Trans Comp Human Interact 5(1):63-108

Vidot N, Cart M, Ferrie J, Suleiman M (2000) Copies convergence in a distributed real-time collaborative environment. In: Proceedings of the 2000 ACM conference on Computer supported cooperative work. ACM Press New York, NY, USA. pp 171-180 